\title{
The BM@N experiment at JINR: status and physics program
}

\section{Mikhail Kapishin}

Joint Institute for Nuclear Research, Laboratory of High Energy Physics, Dubna, Russia E-mail: kapishinejinr.ru

Gleb Pokatashkin *†

Joint Institute for Nuclear Research, Laboratory of High Energy Physics, Dubna, Russia E-mail: pokatejinr.ru

\section{Igor Rufanov}

Joint Institute for Nuclear Research, Laboratory of High Energy Physics, Dubna, Russia E-mail: roufanov@gmail.com

\section{Veronica Vasendina}

Joint Institute for Nuclear Research, Laboratory of High Energy Physics, Dubna, Russia E-mail: vasveronemail.ru

\section{Alexander Zinchenko}

Joint Institute for Nuclear Research, Laboratory of High Energy Physics, Dubna, Russia

E-mail: Alexander.Zinchenkoejinr.ru

BM@N (Baryonic Matter at Nuclotron) is the first experiment to be realized at the accelerator complex of NICA-Nuclotron at JINR (Dubna, Russia). The aim of the experiment is to study interactions of relativistic heavy ion beams with energy up to $4.5 \mathrm{~A} \mathrm{GeV}$ with fixed targets. The research program of the experiment includes studies of strange mesons, multi-strange hyperons and light hyper-nuclei which are produced in nucleus-nucleus collisions close to the kinematic threshold. The BM@N set-up, the experimental program and first results of technical runs are presented.

The European Physical Society Conference on High Energy Physics

5-12 July, 2017

Venice

*Speaker.

†On behalf of the BM@N collaboration 


\section{Introduction}

BM@N (Baryonic Matter at Nuclotron) is the first experiment at the accelerator complex of NICA-Nuclotron. The aim of the BM@N experiment is to study interactions of relativistic heavy ion beams with fixed targets. The Nuclotron heavy ion beam energy range corresponds to $\sqrt{S_{N N}}=2.3-3.5 \mathrm{GeV}$. It is well suited for studies of strange mesons and multi-strange hyperons which are produced in nucleus-nucleus collisions close to the kinematic threshold. The focus of experimental studies will be on hadrons with strangeness, which are produced early in the collision and not present in the initial state of two colliding nuclei. Coalescence of lambda-hyperons with nucleons can produce a variety of light hyper-nuclei [1]. The study of the hyper-nuclei production is expected to provide new insights into the properties of the hyperon-nucleon and hyperon- hyperon interactions. This investigations together with studying productions «cold» nuclear matter in $p p$ and $p A$ reactions give «reference» to pin down nuclear effects.

\section{BM@N set-up}

A sketch of the proposed experimental set-up is shown in Fig. 1. The experiment combines high precision track measurements with time-of-flight information for particle identification and uses total energy measurements for the analysis of the collision centrality.

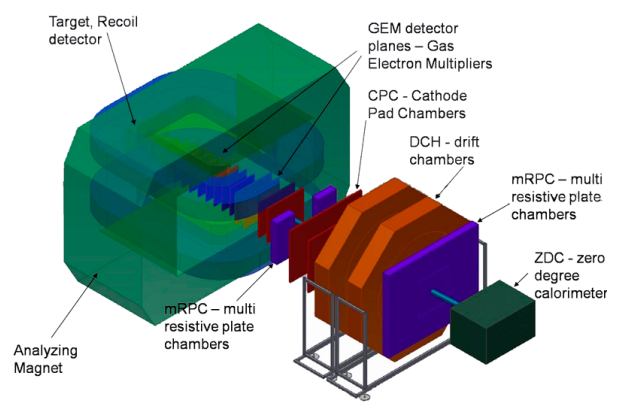

Figure 1: Schematic view of the BM@N set-up.

As a typical fixed-target spectrometer, BM@N contains a dipole analyzing magnet. The gap between the poles of the magnet is around $1 \mathrm{~m}$. The magnetic field can be varied up to $1.2 \mathrm{~T}$ to get the optimal BM@N detector acceptance and momentum resolution for different processes and beam energies.

The central tracker of the BM@N experiment is based on two-coordinate triple GEM detectors [2]. It is located downstream of the target inside the analyzing magnet. The GEM (Gas Electron Multiplier) detectors have the established technology developed at the CERN workshop. They sustain high rates of particles and are operational in the strong magnetic field. The signal recording is done from the anode plane using the double-sided strip readout technique with a strip pitch of $800 \mu$ and a strip orientation of $0^{\circ}$ and $15^{\circ}$ with respect to the vertical axis. The readout board is divided into two zones: the outer and inner ones. The inner (hot) zone with shorter strips will be located near the beam line and is intended to cope with a high particle flux in this region. The drift/cathode pad chambers $(\mathrm{DCH} / \mathrm{CPC})$ of the outer tracker are situated behind the 
magnet. The design parameters of the time-of-flight (ToF) detectors based on multi-gap resistive plate chambers (mRPC) with a strip read-out allow us to discriminate between hadrons $(\pi, K, p)$ as well as light nuclei with the momentum up to few $\mathrm{GeV} / \mathrm{c}$ produced in multi-particle events. The zero degree calorimeter (ZDC) is designed for the analysis of the collision centrality by measuring the energy of forward going particles. The purpose of the trigger detectors, partially covering the backward hemisphere around the target, is to trigger central heavy ion collisions and provide a start time (T0) signal for the mRPC detectors. An electro-magnetic calorimeter (ECAL) is installed behind the outer DCH/CPC chambers and MRPC-2 wall to study processes with electro-magnetic probes $\left(\gamma, e^{+}, e^{-}\right)$in the final state.

\section{Technical run with deuteron beam}

The technical run of the BM@N detector was performed with the deuteron beam in December 2016. The view of the BM@N setup used in the run is presented in Fig. 2 (left). The starting configuration of the central tracker was based on six GEM stations combined from 5 GEM detectors with the size of $66 \times 41 \mathrm{~cm}^{2}$ and 2 GEM detectors with the size of $163 \times 45 \mathrm{~cm}^{2}$. Since the amount of the detectors was quite limited, the tracking stations were arranged to have the beam passing through their centers (Fig. 2 (right)).
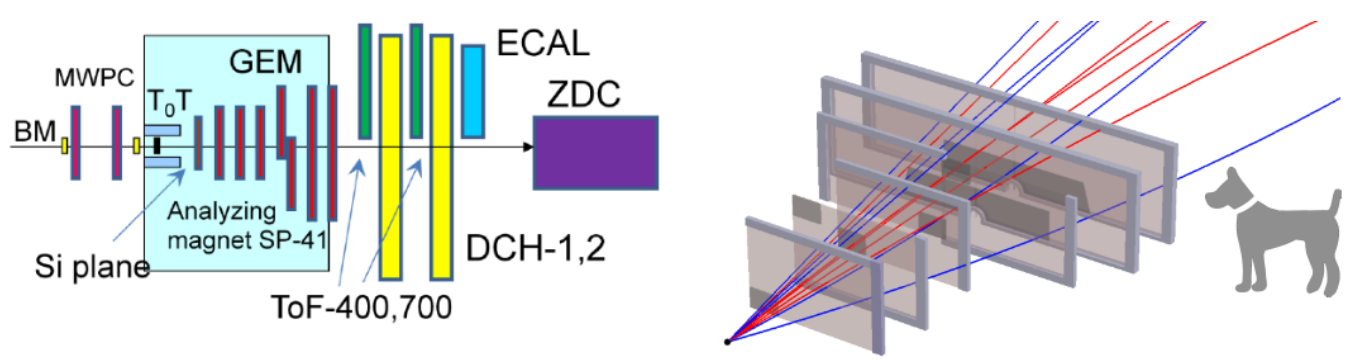

Figure 2: Left) BM@N set-up used in the deuteron run. Right) products of a deuteron-target interaction reconstructed in the central tracker.

The main goal of the technical run was the commissioning of the central GEM tracker and testing and calibration of the other subsystems: ToF, T0 and trigger detectors, ZDC and ECAL calorimeters. The methodical goal of the run was to trace the primary deuteron beam through the set-up with subsequent reconstruction of its momentum at different values of the magnetic field. The reasearch program was devoted to measurements of inelastic reactions $d+$ Target $\rightarrow X$ with the beam kinetic energy of $4 A \mathrm{GeV}$ and different targets: $\mathrm{C}_{2} \mathrm{H}_{4}, \mathrm{C}, \mathrm{Cu}$. Since the GEM tracker configuration was tuned to measure relatively high-momentum beam particles, the geometric acceptance for relatively soft decay products of strange V0-particles was rather low. The Monte Carlo simulation showed that only $\sim 4 \%$ of $\Lambda$ hyperons and $\sim 0.8 \%$ of $K_{s}^{0}$ could be reconstructed.

\section{Results of data analysis}

$\Lambda$-hyperons were reconstructed using their decay mode into two oppositely-charged tracks. The signal event topology (decay of a relatively long-lived particle into two tracks) defined the selection criteria: relatively large distance of the closest approach to the primary vertex of decay 
products, small track-to-track separation in the decay vertex, relatively large decay length of the mother particle. Since particle identification at this stage of the analysis was not used, all positive tracks were considered as protons and all negative as $\pi^{-}$. To reconstruct $\Lambda$-hyperons, 760 thousands events with $\Lambda$-candidates, i.e. containing at least one positive and one negative tracks, were analyzed for 3 targets. The obtained invariant mass distribution of $p \pi^{-}$is shown in Fig. 3 along with the simulated result. One can see a pronounced $\Lambda$-peak.
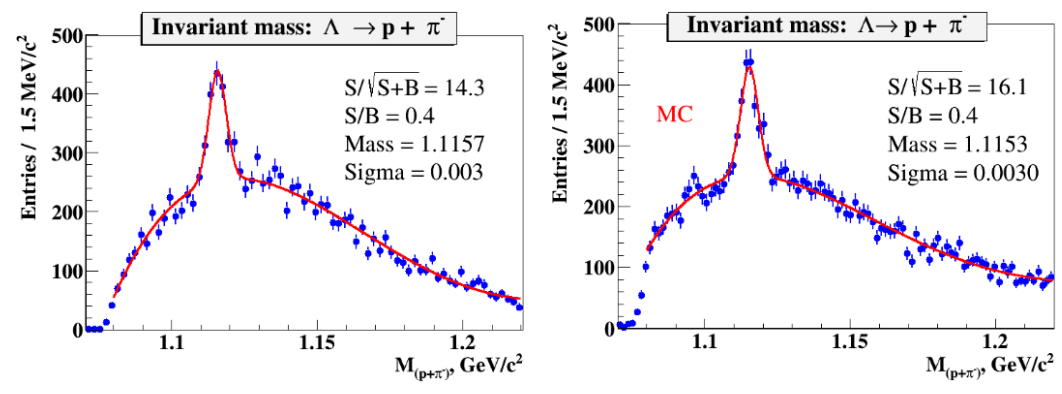

Figure 3: Invariant mass spectrum of $p \pi^{-}$: left) experiment, right) simulation.

\section{Conclusions}

The BM@N experiment is in the starting phase of its operation and has recorded first experimental data. The technical run was performed in the deuteron beam with the kinetic energy of $4 \mathrm{GeV}$ per nucleon. Experimental data of minimum bias interactions of the deuteron beam with different targets were analyzed with the aim to reconstruct tracks, primary and secondary vertexes using the central tracking detectors. The signal of $\Lambda$-hyperon was reconstructed in the invariant mass spectrum of particles originated from secondary vertexes. The reconstructed results were reproduced in realistic Monte Carlo simulation with the identical parameters of the fit. To improve the vertex and momentum resolution and reduce the background under the $\Lambda$-hyperon signal additional planes of the GEM detectors and a set of silicon detectors in front of the GEM tracking detectors will be implemented (Table 1.).

\begin{tabular}{|c|c|c|c|c|c|}
\hline year & 2016 & 2017 spring & 2017 autumn & 2019 & 2020 and later \\
\hline beam & $\mathrm{d}$ & $\mathrm{C}$ & $\mathrm{Ar}, \mathrm{Kr}$ & $\mathrm{Au}$ & $\mathrm{Au}, \mathrm{p}$ \\
\hline max. intensity, $\mathrm{Hz}$ & $1 \mathrm{M}$ & $1 \mathrm{M}$ & $1 \mathrm{M}$ & $1 \mathrm{M}$ & $10 \mathrm{M}$ \\
\hline trigger rate, $\mathrm{Hz}$ & $10 \mathrm{k}$ & $10 \mathrm{k}$ & $20 \mathrm{k}$ & $20 \mathrm{k}$ & $50 \mathrm{k}$ \\
\hline GEM traker status & 6 GEM half pl. & 8 GEM half pl. & 10 GEM half pl. & 8 GEM full pl. & 8 GEM + 4 Si \\
\hline exp. status & tech. run & tech. run & phys. run & stage 1 phys. & stage 2 phys. \\
\hline
\end{tabular}

Table 1: Beam parameters and setup at different stages of the experiment BM@N.

\section{References}

[1] J. Steinheimer et. al, Strangeness at the International Facility for Antiproton and Ion Research, Progress in Particle and Nuclear Physics 62 (2009) 313

[doi:10.1016/j.ppnp.2008.12.037]

[2] D. Baranov et. al (BM@N Collaboration), GEM tracking system of the BM@N experiment, Journal of Instrumentation 12 (C06041) [doi .org/10.1088/1748-0221/12/06/C06041] 